\title{
Liquid-Crystal Phases Formed in Mixed Suspensions of Rod- and Platelike Colloids
}

\author{
F. M. van der Kooij and H. N. W. Lekkerkerker* \\ Van't Hoff Laboratory for Physical and Colloid Chemistry, Debye Institute, Utrecht University, \\ Padualaan 8, $3584 \mathrm{CH}$ Utrecht, The Netherlands
}

Received J une 23, 2000. In Final Form: September 12, 2000

\begin{abstract}
The phase behavior of rod-plate mixtures was investigated using model systems of unambiguously rodand plate-shaped colloids. Neither at equilibrium nor in the early stages of the phase separation process do we observe the theor etically disputed biaxial nematic phase. I nstead, the mixtures show demixing into an isotropic and a separate rod- and a plate-dominated uniaxial nematic phase. The high-concentration regime of the rod-plate phase diagram is exceptionally rich because of the appearance of two extra liquid crystal phases: a rod-rich (presumably) nematic phase and a plate-rich col umnar phase. This leads to the appearance of several four-phase equilibria and even a five-phase equilibrium. We explain the observed topol ogy of the phase diagram by the interplay between the rod- and platelike shape of the particles and their polydispersity.
\end{abstract}

\section{Introduction}

An additional degree of freedom, due to orientation, makes the phase diagram of rod- or platelike colloidal particles intrinsically richer than that of suspensions of spheres. In particular, it may lead to the formation of liquid-crystal phases in which particlesareorientationally ordered. Although perhaps counterintuitive at first thought, such orientational ordering can be explained on purely entropic grounds and may therefore occur in suspensions of colloids that interact through a simplehardbody, that is, short-range repulsive potential. This has been known since Onsager, who demonstrated that although the alignment in a liquid-crystal phase leads to a loss of orientational entropy, it may be compensated by a gain in excluded volume (configurational) entropy if the particle concentration is sufficiently high. ${ }^{1}$ Experimentally, liquid-crystal phases (such as a nematic or smectic) have been observed in colloidal suspensions of inorganic rods (vanadium pentoxide $\left(\mathrm{V}_{2} \mathrm{O}_{5}\right), 2,3$ akaganeite $(\beta$ $\mathrm{FeOOH})^{4}$ and sterically stabilized boehmite $(\mathrm{AlOOH}){ }^{5}$ organic rods (microcrystalline cellulose, ${ }^{6}$ poly(tetrafluoroethylene), ${ }^{7}$ ) and biol ogical rods (Tobacco Mosaic virus ${ }^{8-10}$ and fd virus ${ }^{11}$ ), amply demonstrating the validity of Onsager's theory in thecase of rodlikeparticles. For plateshaped colloids, a model system displaying a comparably rich phase behavior has been devel oped only recently. This system of sterical ly stabilized gi bbsite $\left(\mathrm{Al}(\mathrm{OH})_{3}\right)$ platel ets exhibits an isotropic, a nematic, ${ }^{12}$ and a col umnar phase, ${ }^{13}$ respectively, upon increasing the particle concentration.

(1) Onsager, L. Ann. N. Y. Acad. Sci. 1949, 51, 627

(2) Zocher, H. Anorg. Allg. Chem. 1925, 147, 91

(3) Pelletier, O.; Davidson, P.; Bourgaux, C.; Livage, J . Europhys. Lett. 1999, 48, 53.

(4) Maeda, Y.; Hachisu, S. Colloids Surf. 1983, 7, 357.

(5) van Bruggen, M. P. B.; Dhont, J . K. G.; Lekkerkerker, H. N. W . Macromolecules 1999, 32, 2256.

(6) Dong, X. M.; Kimura, T.; Revol , J . F .; Gray, D. G. Langmuir 1996 12, 2076. 55.

(7) Folda, T.; Hoffmann, H.; Chanzy, H.; Smith, P. Nature1988, 333,

(8) Bernal, J. . D.; Fankuchen, I. J . Gen. Physiol. 1941, 25, 111.

(9) Kreibig, U.; Wetter, C. Z. Naturforsch. 1980, 35, 750.

(10) Fraden, S.; Hurd, A. J .; Meyer, R. B.; Cahoon, M.; Caspar, D.

L. D. J . Physique 1985, 46.

(11) Dogic, Z.; Fraden, S. Phys. Rev. Lett. 1997, 78, 2417.

(12) van der Kooij, F. M.; Lekkerkerker, H. N. W. J . Phys. Chem. B $1998,102,7829$.
The phase behavior of mixtures of rod- and platelike particles is not only increasingly richer than that of rods and plates separately but al so far less understood. In the discussion on the rod-plate phase diagram a central role is played by the stability of a biaxial nematic phase. In a biaxial phase both the rodlike and the platelike species are orientationally ordered, the axis of alignment of the rods being perpendicular to that of the plates. Alternatively, this biaxial phase may be unstable to demixing into two separate uniaxial nematic phases containing predominantly rodsand plates, respectively. Thedifficulty in predicting the phase behavior of rod-platemixtures in theory ${ }^{14-18}$ and simulation ${ }^{19}$ originates from the subtle balance between the mixtures' three sources of entropy, due to orientation, excluded volume, and mixing. In experiments, evidencefor biaxiality in rod-platemixtures was originally thought to exist in a system of micelles. ${ }^{20}$ The observed biaxial phase was later reinterpreted however as the act of a single micellar species, its shape being either intrinsically biaxial or changing across the transition. ${ }^{21}$

In the present study weinvestigate the phase behavior of a mixture of rods and plates that are unambiguously rod- and plate-shaped. This mixture is a combination of theabove-mentioned model systems of sterically stabilized boehmite rods ${ }^{5}$ and gibbsite platel ets. ${ }^{12} \mathrm{~F}$ irst, we employ this rod-plate mixture to pursue the issue of biaxial nematicstability ver sus demixing in two uniaxial nematic phases. Preliminary results ${ }^{22}$ are extended here to cover both the kinetic pathway of the phase separation as well as the final equilibrium states. Moreover, wefocus on the

(13) van der Kooij, F. M.; Kassapidou, K.; Lekkerkerker, H. N. W Nature 2000, 406, 868

(14) Stroobants, A.; Lekkerkerker, H. N. W.J . Phys. Chem. 1984, 88, 3669

(15) Alben, R. J . Chem. Phys. 1973, 59, 4299

(16) Rabin, Y.; Mullen, W. E. M.; Gelbart, W. M. Mol. Cryst. Liq. Cryst. 1982, 89, 67.

(17) Chrzanowska, A. Phys. Rev. A 1998, 58, 3229.

(18) van Roij, R.; Mulder, B. J . Phys. (France) II 1994, 4, 1763.

(19) Camp, P.; Allen, M. P.; Bolhuis, P. G.; Frenkel, D. J . Chem. Phys. 1997, 106, 9270.

(20) Yu, L. J .; Saupe, A. Phys. Rev. Lett. 1980, 45, 1000. 8036 .

, E. Fenriques, V.B. J. Chem. Phys. 1997, 107,

(22) van der Kooij, F. M.; Lekkerkerker, H. N. W. Phys. Rev. Lett. 2000, 84, 781. 
Table 1. Particle Characterization Results for the Rods and Plates Used in This Study (Given Sizes Reflect the Dimensions of the Grafted Particles, Obtained by Adding Twice the Estimated Polymer Layer Thickness to the TEM Dimensions)

\begin{tabular}{lccc}
\hline & long axis $(\mathrm{nm})$ & short axis $(\mathrm{nm})$ & aspect ratio \\
\hline rods & $186( \pm 30 \%)$ & $18( \pm 30 \%)$ & 10 \\
plates & $208( \pm 25 \%)$ & $14( \pm 20 \%)$ & 15
\end{tabular}

rod-plate phasediagram at high particleconcentrations. While, so far, theoretical efforts were directed toward the biaxial versus demixing issue, the possibility that rodplate mixtures may form higher order liquid crystals has not yet been considered. This possibility is investigated in the high-concentration regime.

\section{Experimental Section}

2.1. Synthesis and Characterization of the Model System. The employed model systems of rod- and platelike colloids consist of sterically stabilized boehmite $(\mathrm{AIOOH})$ and gibbsite $\left(\mathrm{AL}(\mathrm{OH})_{3}\right)$ particles, respectively, dispersed in toluene. Theinitial aqueous suspensions are prepared by hydrothermal treatment (at 150 and $85^{\circ} \mathrm{C}$, respectively) of $0.09 \mathrm{M} \mathrm{HCl}$ solutions containing both $0.08 \mathrm{M}$ aluminum tri-sec-butoxide and $0.08 \mathrm{M}$ aluminum tri-iso-propoxide. ${ }^{22-24,12}$ Both systems are subsequently grafted with a $\left(M_{n} \approx 1000\right)$ modified polyisobutylene ${ }^{25,12}$ to provide the particles with an approximately hard-body interaction potential. The thickness of this polymer layer has been estimated at 4 $\mathrm{nm} .{ }^{26}$

Particle size distributions of rods and plates are determined from transmission electron microscopy (TEM) images. The estimated thickness of the polymer layer is added tothenumberaverage TEM dimensions to obtain the size of the grafted particles, shown in Table 1. The polydispersity in particle size is defined as the relative standard deviation $\sigma_{\mathrm{x}}=\sqrt{\left\langle\mathrm{x}^{2}\right\rangle-\langle\mathrm{x}\rangle^{2}} /\langle\mathrm{x}\rangle$, where $\mathrm{x}$ is the corresponding dimension of the rod or plate. In the case of the gibbsite platelets, the particle diameter is determined as the circle with an area equal to that of the approximately hexagonal particle. Prior to the examination of the thickness of the platelets by TEM, some of the gibbsite suspension is deliberately flocculated by the addition of water to facilitate edgewise imaging.

To study the phase diagram of mixtures of these systems, weighed amounts of stock suspensions of rods and plates are homogenized in 2-mm cuvettes and stored under thermostatic conditions to reach equilibrium. The colloid volume fraction $\phi$, which includes the solvent present in the grafted polymer layer, is obtained by dividing the mass concentration c (determined by drying a known amount of dispersion at $75^{\circ} \mathrm{C}$ to constant weight) by the effective mass density $\rho_{\text {eff }}$ as described in refs 5,27 , and 28. For the rod-and platelike particles we have $\rho_{\text {eff }}=1.2 \mathrm{~g} / \mathrm{cm}^{3}$ and $\rho_{\text {eff }} 1.3 \mathrm{~g} / \mathrm{cm}^{3}$, respectively, each with an estimated error of $10 \%$ due to the uncertainty in polymer layer thickness.

2.2. Scattering and Microscopy Experiments. Small-angle $X$-ray scattering (SAXS) experiments were performed on the DUBBLE beam line at the ESRF, Grenoble, France. The X-ray beam is highly monochromatic $\left(\Delta \lambda / \lambda \approx 2 \times 10^{-4}\right)$ and has a narrow focus (about $0.5 \times 0.5 \mathrm{~mm}^{2}$ ). The wavelength was fixed at 0.138 $\mathrm{nm}$. A $512 \times 512$ pixel detector was placed at about $8.5 \mathrm{~m}$ from the sample, yielding an accessible q range of about $0.02-0.7$ $\mathrm{nm}^{-1}$. Theq axis is cal ibrated usingan oriented hydrated col lagen fiber of a rat tail tendon. The raw two-dimensional diffraction

(23) Philipse, A. P.; Nechifor, A. M.; Patmamanoharan, C. Langmuir 1994, 10, 4451.

(24) Wierenga, A.; Lenstra, T. A. J .; Philipse, A. P. Colloids Surf. A 1998, 134, 359.

(25) Buining, P. A.; Veldhuizen, Y. S. J .; Pathmamanoharan, C.; Lekkerkerker, H. N. W. Colloids Surf. 1992, 64, 47.

(26) Smits, C.; Briels, W. J .; Dhont, J . K. G.; Lekkerkerker, H. N. W. Prog. Colloid. Polym. Sci. 1989, 79, 287.

(27) van der Kooij, F. M.; Boek, E. S.; Philipse, A. P., submitted to J. Colloid Interface Sci. 2000.

(28) van der Kooij, F. M.; Vogel, M.; Lekkerkerker, H. N. W., Phys. Rev. E 2000, 62, 5397.

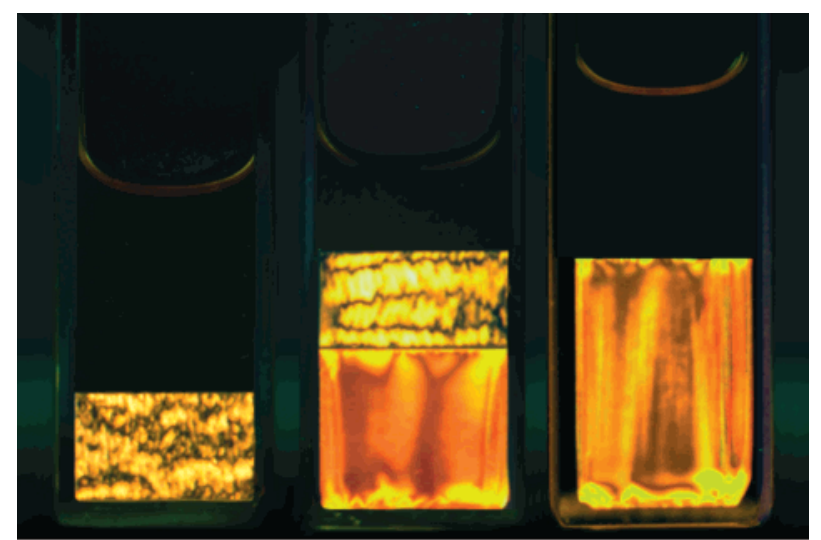

(a)

(b)

(c)

Figure 1. Phase separation in moderately concentrated rodplate mixtures, as observed between crossed polarizers. Depicted are (a) an isotropic upper phase in coexistence with a nematic $\mathrm{N}^{+}$bottom phase, (b) I $+\mathrm{N}^{+}+\mathrm{N}^{-}$coexistence, and (c) I $+\mathrm{N}^{-}$coexistence. The overall compositions of the samples range from (a) $\left(\phi_{\text {rod }}, \phi_{\text {plate }}\right)=(0.10,0.01)$, (b) $(0.10,0.08)$, to (c) $(0.02,0.18)$

Table 2. Rod/Plate Number Ratios $\mathbf{N}_{\text {rod }} / \mathbf{N}_{\text {plate }}$ in the Coexisting Phases after Phase Separation, as Determined from TEM Micrographs (Corresponding Number Ratios in the Samples before Demixing Are Included for Comparison)

\begin{tabular}{|c|c|c|c|c|c|c|c|}
\hline \multirow[b]{2}{*}{ sample } & \multicolumn{2}{|c|}{ overall composition } & \multicolumn{5}{|c|}{$\mathrm{N}_{\text {rod }} / \mathrm{N}_{\text {plate }}$} \\
\hline & $\left(\phi_{\text {rod }}, \phi_{\text {plate }}\right)$ & $\mathrm{N}_{\text {rod }} / \mathrm{N}_{\text {plate }}{ }^{\mathrm{a}}$ & 1 & $\mathrm{~N}^{+}$ & $x$ & $\mathrm{~N}^{-}$ & $\mathrm{C}$ \\
\hline $\begin{array}{l}+\mathrm{N}^{+}+\mathrm{N}^{-} \\
\mathrm{J}^{+}+\mathrm{N}^{-}+\mathrm{C} \\
\mathrm{J}^{+}+\mathrm{X}\end{array}$ & $\begin{array}{l}(0.10,0.08) \\
(0.05,0.31) \\
(0.17,0.05)\end{array}$ & $\begin{array}{c}13 \\
2.2 \\
34\end{array}$ & 39 & $\begin{array}{l}26 \\
21 \\
68^{b}\end{array}$ & $45^{b}$ & $\begin{array}{l}2.7 \\
0.7\end{array}$ & 0 \\
\hline
\end{tabular}

a Calculated from therod/platevolumefraction ratio ( $\left.\phi_{\text {rod, }}, \phi_{\text {plate }}\right)$ using the particle volume ratio $v_{\text {rod }} / v_{\text {plate }} \approx 1 / 10$ as estimated from the grafted particle dimensions (Table 1 ). ${ }^{\text {b }}$ Number ratios are determined by counting several hundreds of particles. N evertheless, statistics for samples with very large rod/plate number ratios are insufficient to provide quantitative agreement with the corre sponding overall value estimated from the rod/plate volume fractions.

patterns are radially averaged after correction for transmission and background radiation. Prior totheSAXS experiment, samples were stored in a thermostated room for 2 weeks to reach phase equilibrium.

Samples studied by polarization microscopy were prepared in flat, 0.2-mm path length capillaries that weresubsequently sealed with an epoxy glue. Prior to the microscopy experiment, the samples are homogenized by ultrasonic treatment for a few minutes.

\section{Results}

3.1. Phase Behavior at Moderate Concentration. Up to moderate volume fractions $\left(\phi_{\text {total }} \lesssim 0.3\right.$ ) the rodplate phase diagram comprises three regions of phase coexistence (Figure 1). For rod-rich mixtures, phase separation yiel ds an isotropic phase (I) in coexistence with a nematic phase $\left(\mathrm{N}^{+}\right)$of predominantly rods. Plate-rich samples, on the other hand, show coexistence of an isotropic and a nematic phase $\left(\mathrm{N}^{-}\right)$containing mostly plates. The plus and minus signs used to denote these nematics refer to the axis of orientational order, which is either parallel (in the case of the rodlike $\mathrm{N}^{+}$phase) or perpendicular (for the platelikeN- phase) tothedirection of the particles' highest refractive index. I $+\mathrm{N}^{+}+\mathrm{N}^{-}$ triple coexistence is observed over the broad range of intermediate rod-plate compositions. TEM micrographs confirm the rod- and plate-dominated nature of the $\mathrm{N}^{+}$ 
Table 3. Average Diameter (nm) and Polydispersity of the Platelets in the Coexisting Phases That Appear in Table 2

\begin{tabular}{cccccc}
\hline sample & $\mathrm{I}$ & $\mathrm{N}^{+}$ & $\mathrm{X}$ & $\mathrm{N}^{-}$ & $\mathrm{C}$ \\
\hline $\mathrm{I}+\mathrm{N}^{+}+\mathrm{N}^{-}$ & $184( \pm 25 \%)$ & $205( \pm 24 \%)$ & & $268( \pm 19 \%)$ & $238( \pm 26 \%)$ \\
$\mathrm{N}^{+}+\mathrm{N}^{-}+\mathrm{C}$ & & $138( \pm 33 \%)$ & & $221( \pm 25 \%)$ & \\
$\mathrm{N}^{+}+\mathrm{X}$ & & $187( \pm 23 \%)$ & $2217 \%)$
\end{tabular}

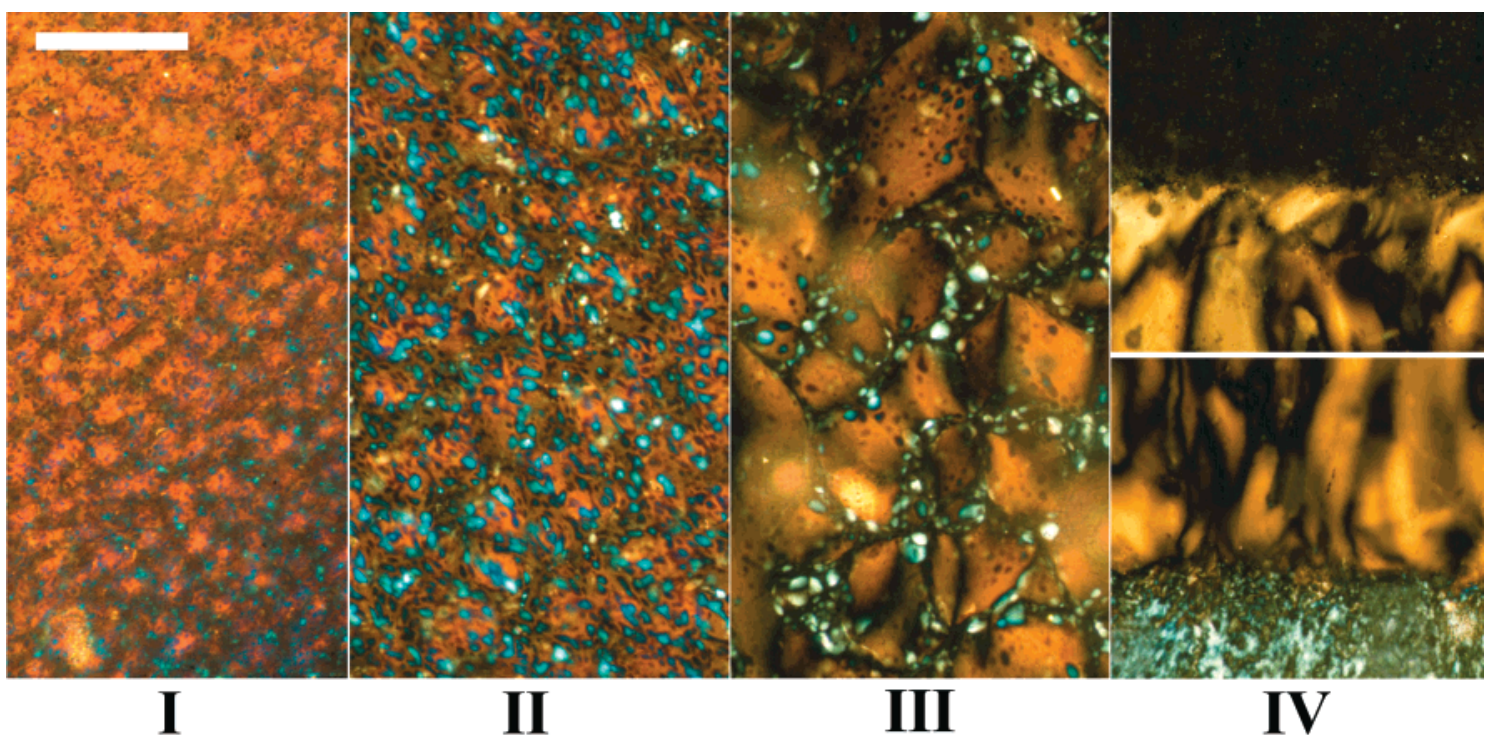

Figure 2. The course of the phase separation process in the I $+\mathrm{N}^{+}+\mathrm{N}^{-}$triple coexistence region, as observed by polarization microscopy. The stages I-IV correspond to $30 \mathrm{~min}, 4 \mathrm{~h}, 20 \mathrm{~h}$, and 2 days after homogenizing the sample. Stage IV depicts the I $-\mathrm{N}^{+}$interface (top image) and the $\mathrm{N}^{+}-\mathrm{N}^{-}$interface (bottom image) after macroscopic phase separation in a vertically oriented sample. The composition of the studied rod-plate mixture is $\left(\phi_{\text {rod, }} \phi_{\text {plate }}\right)=(0.06,0.13)$. The size represented by the scale bar varies from $200 \mu \mathrm{m}$ in stages I and II to $800 \mu \mathrm{m}$ in stages III and IV.

and $\mathrm{N}^{-}$phase, respectively, with subsequent rod/plate number density ratios being presented in Table 2 . Along with the segregation of the rod and plate populations in the coexisting $+\mathrm{N}^{+}+\mathrm{N}^{-}$phases, TEM mi crographsal so demonstrate that pronounced fractionation with respect to size of the particles has occurred. For instance, the diameter of the platelets in the plate-dominated nematic $\mathrm{N}^{-}$is roughly $40 \%$ larger than that in the coexisting I and $\mathrm{N}^{+}$phase while, at the same time, the polydispersity in the $\mathrm{N}^{-}$phase is reduced significantly below that of the parent suspension (see Table 3).

The biaxial nematic phase, which is not observed in the moderate concentration regime nor in any other part of the phase diagram, is apparently not stable for the rodplate mixture studied here. One may wonder, however, if anal ogous totherole of a metastablegas-liquid bi nodal 29 in the fluid-crystal transition kinetics of hard-sphere suspensions the biaxial phase is involved in the I $+\mathrm{N}^{+}$ $+\mathrm{N}^{-}$demixing process as a metastable intermediate. Moreover, the evolution of demixing in the triple coexistence region is an issue of fundamental interest, which has been studied earlier for a mixture of colloidal spheres and polymer undergoing gas-liquid-crystal separation. ${ }^{30,31}$ Wetherefore examinethekinetic pathway of the $\mathrm{I}+\mathrm{N}^{+}+\mathrm{N}^{-}$phase separation, using polarization microscopy. The early stages of the phase separation, illustrated by Figure 2, involve the formation of two distinct liquid-crystal phases, which simultaneousl y evolve but by different mechanisms. By comparison to the final state (Figure 2, stage IV) we identify the yellowish birefringent domains as the rodlike nematic $\mathrm{N}^{+}$and the

(29) Illet, S. M.; Orrock, A.; Poon, W. C. K.; Pusey, P. N. Phys. Rev. E 1995, 51, 1344.

(30) Poon, W. C. K.; Renth, F.; Evans, R. M. L.; Fairhurst, D. J .; Cates, M. E.; Pusey, P. N. Phys. Rev. Lett. 1999, 83, 1239.

(31) Poon, W. C. K.; Renth, F.; Evans, R. M. L. J . Phys.: Condens. Matter 2000, 12. bluish domainsas the platelikenematic $\mathrm{N}^{-}$. Theformation of the $\mathrm{N}^{+}$phase proceeds by the mechanism of spinodal decompositi on, ${ }^{5}$ its spinodal density fluctuations growing in amplitudeand wavelength with time. At thesametime, and apparently independent from the emerging $\mathrm{N}^{+}$phase, a platelike nematic $\mathrm{N}^{-}$phase forms by the mechanism of nucleation and growth of the corresponding nematic droplets. Under the microscope, the onset of $\mathrm{N}^{+}-\mathrm{N}^{-}$ demixing can be observed al ready at 5 min after homogenization of thesample. Themicroscopic observations thus demonstratethat, from the very early stages on, the phase separation involves demixing of the two uniaxial nematics rather than the formation of a biaxial nematic phase.

3.2. Phase Behavior at High Concentration. At high volume fractions of rods and plates $\left(0.3 \lessgtr \phi_{\text {total }} \leqslant 0.5\right)$ the phase behavior becomes increasingly rich. In principle, one would expect the isotropic phase to vanish if the concentration of a I $+\mathrm{N}^{+}+\mathrm{N}^{-}$sample is raised. I nstead, increasing both the rod and the plate concentration leads to the appearance of one, and eventually two extra liquidcrystal phases (Figure 3). TEM images demonstrate that one of theseextra phases contains al most exclusivel y rods whiletheother is explicitly plate-rich (Table2). Thenature of the rod-rich phase has not been established unambiguously yet. A preliminary SAXS measurement on this phase (which we denoteby X) shows that its scattering is typical for a nematic, rather than a smectic phase, as it lacks scattering peaks in the range of $q \approx 2 \pi /$ (rod length). The SAXS measurement is not conclusive, however, as the amount of $X$ phase available for the experiment was very small such that some solvent may have evaporated.

TheSAXS pattern of theother denseliquid-crystal phase (Figure 4 curve b), comprising predominantly plates, reveals that this is a columnar phase $(C)$. The structure characterizing a columnar phase is a regular twodimensional lattice of columns, the columns being one- 


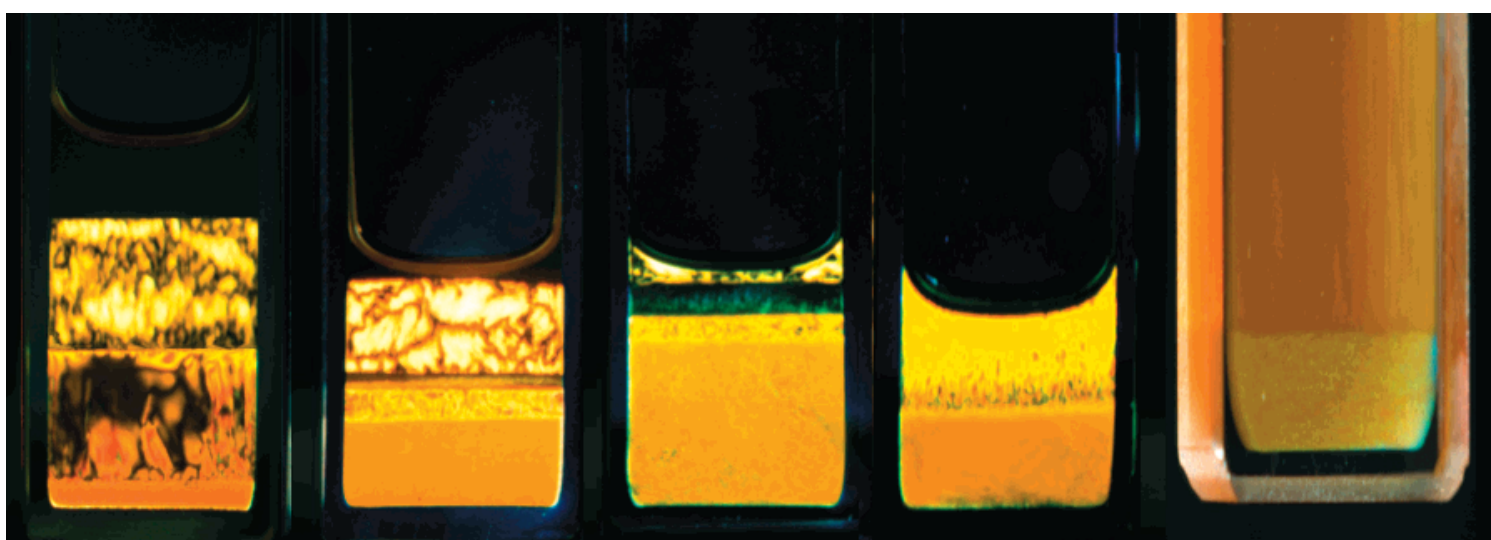

(a)

$(b)$

$(c)$

(d)

$(e)$

Figure 3. Phase-separated rod-plate mixtures as observed between crossed polarizers. Depicted are (a) four-phase I $+\mathrm{N}^{+}+\mathrm{N}^{-}$ $+\mathrm{C}$ coexistence (denoted in order from top to bottom) in a sample with the composition ( $\left.\phi_{\text {rod, }} \phi_{\text {plate }}\right)=(0.06,0.26)$, (b) five-phase $\mathrm{I}+\mathrm{N}^{+}+\mathrm{X}+\mathrm{N}^{-}+\mathrm{C}$ coexistence at $(0.10,0.22)$, (c) four-phase $\mathrm{N}^{+}+\mathrm{X}+\mathrm{N}^{-}+\mathrm{C}$ coexistence at $(0.07,0.29)$, and (d) two-phase $\mathrm{N}^{-}$ $+C$ coexistence at $(0.03,0.35)$. I mage (e) depicts a sampleat $(0.02,0.35)$ exhibiting $N^{-}+C$ coexistence, photographed without crossed polarizers but illuminated by white light to demonstrate the Bragg reflections in the lower (C) phase.

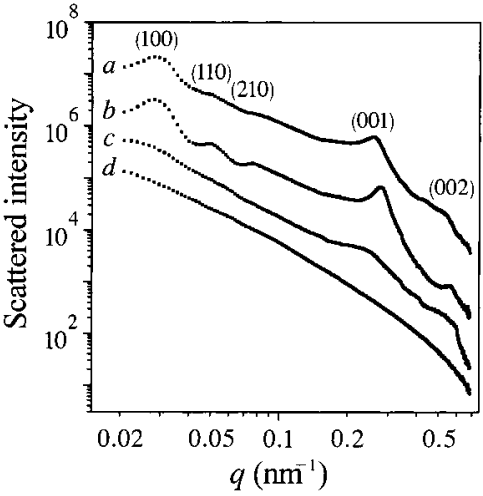

Figure 4. SAXS pattern of (a) the columnar phase in a plate suspension with an overall concentration $\phi_{\text {plate }}=0.45$, (b) the columnar phase in a rod-plate mixture of overall composition $\left(\phi_{\text {rod }}, \phi_{\text {plate }}\right)=(0.02,0.35)$, (c) nematic phase that coexists with the col umnar phase depicted by curve (b), and (d) a very dilute suspension of plates ( $\phi_{\text {plate }} \approx 0.01$ ) reflecting the form factor of the platelets. Scattered intensities are in arbitrary units and curves are shifted vertically for clarity.

Table 4. q Values of the Peak Maxima as They Appear in the SAXS Patterns of the Columnar Phase in a Pure Plate Suspension and a Rod-Plate Mixture ${ }^{a}$

\begin{tabular}{llllll}
\hline & \multicolumn{5}{c}{$\mathrm{q}\left(10^{-2} \mathrm{~nm}^{-1}\right)$} \\
\cline { 2 - 6 } & $(100)$ & $(110)$ & $(210)$ & $(001)$ & $(002)$ \\
\hline a & 2.89 & 5.0 & 7.4 & 25.6 & \\
b & 2.81 & 4.99 & 7.51 & 27.8 & 56
\end{tabular}

a The overall composition of samples $\mathrm{a}$ and $\mathrm{b}$ is given by ( $\phi_{\mathrm{rod}}$, $\left.\phi_{\text {plate }}\right)=(0,0.45)$ and $(0.02,0.35)$, respectively.

dimensional liquidlikestacks of platelets. The scattering of the columnar phase is readily distinguished from a nematic $\mathrm{N}^{-}$phaseand an isotropicplatesuspension, which are depicted by curves $\mathrm{c}$ and $\mathrm{d}$ in Figure 4 . In the small q regime, wherethespacing $d=2 \pi / q$ is of the order of the diameter of the platelets, the columnar phase exhibits one major peak and one or perhaps two additional peaks. The q values of these peaks (Table 4), whoseq ratio is like $1: \sqrt{ } 3: \sqrt{ } 7$, demonstrates that ordering in the plane of the platediameter is hexagonal with thepeaks corresponding to the (100), (110), and (210) reflections. The two peaks at much larger q correspond to 1 and 0.5 times a spacing of roughly theplatethickness. Wether eforeidentify them as the (001) and (002) reflections of the liquidlike order between the plates along the column axis. The columnar signature of this phase, as opposed to a hexagonally ordered layerlikestructurethat would giverisetosimilar peaks, is demonstrated by unaffected $q$ values of the scattering peaks upon tilting a sample in a flat capillary with respect to the X-ray beam. $32,33,13$ A crystalline arrangement of the particles is not explicitly ruled out by theobservations, although this possibility is most unlikely.

In Figure 4, scattering from the columnar phase in a rod-plate mixture (curve b) is compared to the columnar phase in the absence of rods (curve a). Despite the considerable concentration of rods in the first (Table 2 ), theSAXS patterns are notably similar, in particular, with respect to the position of the low q peaks reflecting the hexagonal ordering between columns. The (001) peak, corresponding to the liquidlike order along the $z$ axis of the col umn, is shifted to slightly larger q in the case of the rod-platemixture, suggesting that thestructureis either slightly compressed or that the $C$ phasecontains relatively thin platel ets as the result of fractionation. As the length of the rods is almost equal to the plate diameter, the entropically most favorable location of the rods appears to be between the plates in the column, their long axis being oriented perpendicular to the column axis. From themeasured scattering patterns it cannot beestablished whether this is the actual arrangement of the rods, but this picture does not conflict with the SAXS patterns either. A prominent effect of the presence of rods in the columnar phase is that, judged by the intensity of the scattering peaks, it makes columnar ordering more pronounced. I n an earlier study whereplatelet suspensions without rods were considered, a similar enhancement of columnar ordering was observed when the polydispersity in platel et diameter was reduced. As discussed bel ow, the reduction of polydispersity due to fractionation is particularly strong in the $C$ phase of the rod-plate mixture, which may explain the more pronounced columnar ordering manifested by its SAXS pattern.

The emergence of the liquid-crystalline $C$ and $X$ phases and their subsequent conjunction with the $\mathrm{I}^{+} \mathrm{N}^{+}$, and $\mathrm{N}^{-}$ phases coexistence regions makes the overall topology of the rod-plate phase diagram notably rich (Figures 3 and

(32) Guinier, A. X-ray Diffraction in Crystals, Imperfect Crystals, and Amorphous Bodies; Dover: New York, 1994.

(33) Brown, A. B. D.; Ferrero, C.; Narayanan, T.; Rennie, A. R. Eur. Phys. J. B 1999, 11, 481. 


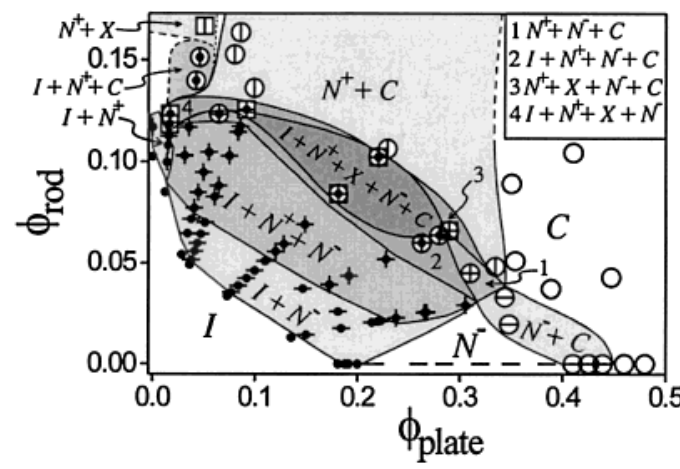

Figure 5. Experimental phase diagram of the rod-plate mixtures. Symbols $(\mathrm{I}), \mid\left(\mathrm{N}^{+}\right),-\left(\mathrm{N}^{-}\right), \square(\mathrm{X})$, and $\mathrm{O}(\mathrm{C})$ indicate which phases were observed at the studied points in the phase diagram. Boundaries of the phaseregions areindicated by lines, their shapeand positioning based on thedata pointsthey enclose and on the consistency with surrounding phase regions. Lines are dashed in regions where the limited number of data points does not allow precise location of the phase boundaries.

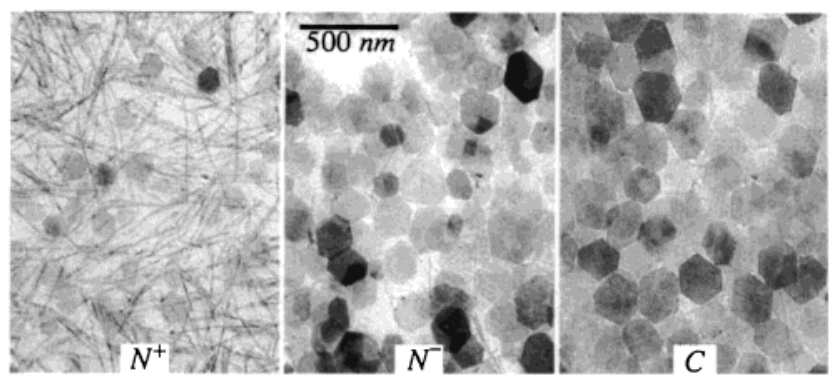

Figure 6. TEM micrographs of each of the $\mathrm{N}^{+}, \mathrm{N}^{-}$, and $\mathrm{C}$ phases at triple coexistence.

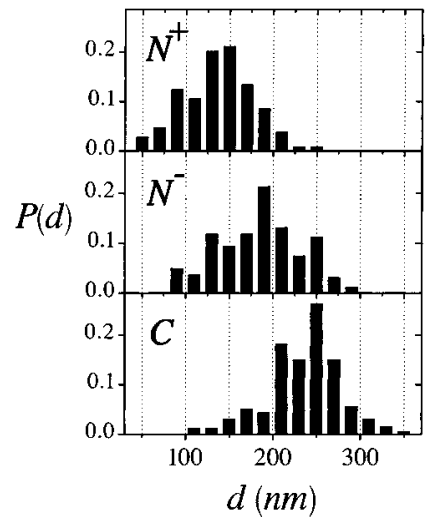

Figure 7. Diameter distributions $P(D)$ in thecoexisting phases at $\mathrm{N}^{+}-\mathrm{N}^{-}-\mathrm{C}$ equilibrium, as determined from TEM micrographs. The distributions are based on around 150 particles each and normalized such that $\sum P\left(D_{i}\right)=1$.

5). A variety of two-, three-, and four-phase equili bria and even a five-phase equilibrium is observed, each of these phase regions being based on combinations of the $\mathrm{I}, \mathrm{N}^{+}$, $\mathrm{N}^{-}, \mathrm{C}$, and $\mathrm{X}$ phases. The reason for the diversity of the phase diagrams and the observed topology is discussed in the next section.

Fractionation of the platediameter in the $X$ and $C$ phase is studied for samples at $\mathrm{N}^{+}+\mathrm{X}$ and $\mathrm{N}^{+}+\mathrm{N}^{-}+\mathrm{C}$ coexistence (see Table 3 and Figures 6 and 7). In the $X$ phase, reduction of the platelets' polydispersity by fractionation is weak, which is not surprising for a roddominated nematic phase that coexists with another rodrich nematic $\mathrm{N}^{+}$. In thecol umnar phase, on theother hand, $\sigma_{\mathrm{D}}$ is reduced to $17 \%$ for the studied mixture at triple coexistence. The columnar phase in a rod-plate mixture is hence very effective in reducing the polydispersity of the platel ets. In this respect, it surpasses the $C$ phase in theabsence of rods, ${ }^{13}$ the $\mathrm{N}^{-}$phasein a rod-platemixture (previous section), the $\mathrm{N}^{-}$phase in plate-polymer mixtures, ${ }^{28}$ and particularly the $\mathrm{N}^{-}$phase in pure plate suspensions. ${ }^{12}$

\section{Discussion}

The coexistence of up to five phases, as observed in concentrated rod-platemixtures, prompts us toreconsider the perception of the experimental system as a simple rod-platemixture. This is due to the phase rule of Gibbs, which implies that, at a given temperature, five-phase coexistencein a colloidal suspension requires the presence of at least four different colloidal species. I $\mathrm{n}$ reality, both therod and platesuspensions are polydisperse and hence represented by a virtually infinite number of species differing in size. Here, we will use the fact that because of the competing costs of mixing entropy, the number of coexisting phases is limited to a maximum of five, which allows us to represent the polydisperse rod-platemixture by four effective components. This facilitates understanding of the gl obal features of the observed phase diagram. Led by the rod- or plate-dominated nature of the four different liquid-crystal phases that appear in the phase diagram, weassign two of the effectivecomponents totwo monodisperse rodlike species and the two remaining components to two monodisperse platelike species. The difference between thelikespecies is that they are unequal in size. The high-dimensional rod-plate phase space is now projected onto a four-dimensional phase diagram, set up by four axes that correspond to the concentrations of the four different components. The most eye-catching feature of theexperimental phasediagram, the five-phase $\mathrm{I}+\mathrm{N}^{+}+\mathrm{N}^{-}+\mathrm{X}+\mathrm{C}$ coexistence region, appears in the four-dimensional phase diagram in the region wherefive four-phase regions, comprising five different four-phase combinations of the $, \mathrm{N}^{+}, \mathrm{N}^{-}, \mathrm{X}$, and $\mathrm{C}$ phases, intersect. These, in turn, are surrounded by a total number of 5!/ $(3 ! 2 !)=10$ different three-phase coexistence regions, 5!/ $(2 ! 3 !)=10$ two-phase regions, and finally the $5 ! /(1 ! 4 !)=$ 5 single-phase regions $\left(\mathrm{I}, \mathrm{N}^{+}, \mathrm{N}^{-}, \mathrm{X}\right.$, and $\mathrm{C}$ ). In the experiment we can vary only two parameters, the concentration of the polydisperse rods and that of the polydisperse plates. The experimentally accessible rodplate mixtures therefore lie in one plane of rod vs plate concentration. This two-dimensional experimental plane cuts through the four-dimensional phase diagram, apparently with an angle such that it hits the five-phase coexistenceregion. This explains why, in the experiment, we observe a five-phase equilibrium and several four-, three, two-, and single-phase equilibria, whileat thesame time a few phase equilibria are absent. That is because, geometrically, the two-dimensional experimental plane simply cannot cut through all phase regions that are present in thefour-dimensional rod-platephasediagram. The polydisperse system's ability to follow the entropic pathway toward multiphase coexistence, rather than to end in glassy structures, may neverthel ess be considered a remarkable feature.

\section{Conclusions}

We have studied the phase behavior of the first experimental system consisting of unambiguously rodand plate-shaped particles. Neither at equilibrium nor in the early stages of the phase separation process do we observe thetheoretically disputed biaxial nematic phase. Instead, the mixtures show demixing into an isotropic (I) 
and a separate rod- and a plate-dominated uniaxial nematic phase $\left(\mathrm{N}^{+}\right.$and $\left.\mathrm{N}^{-}\right)$. Thehigh-concentration regime of therod-platephasediagram comprises twoextraliquidcrystal phases, being a rod-rich (presumably) nematic phase $X$ and a plate-rich col umnar phase C. By conjunction with the $\mathrm{I}, \mathrm{N}^{+}$, and $\mathrm{N}^{-}$coexistence regions, the $\mathrm{X}$ and $\mathrm{C}$ phases lead to the appearance of three four-phase equilibria and even a five-phase equilibrium. Understanding a similar topology of the phase diagram requires that the rod- and platelike shapes of the particles as well as their polydispersity betaken into account. Thechallengehence raised for theoretical studies and computer simulations is 2-fold: to investigatethe phasebehavior of monodisperse rod-plate mixtures at high concentrations to establish the stability of higher density liquid-crystal phases (columnar, smectic, or perhaps a mixed phase) and, second, to address the effect of polydispersity on the rod-plate phase diagram.

Acknowledgment. We thank $K$. Kassapidou for assistance with the SAXS experiments and I. Dolbnya and W. Bras for technical support at DUBBLE. This work was supported by the Foundation for Fundamental Research on Matter (FOM) and The N etherlands Organization for the Advancement of Research (NWO).

LA000875R 